\title{
High Efficiency Wireless Power Transfer System for Medium Power
}

\author{
Zhiyuan Shi, Shaorong Fang, Nenggui Chen and Zuosheng Xie* \\ Xiamen University, Xiamen, China \\ *Corresponding author
}

\begin{abstract}
As the technology matures and increased demand for wireless charging, the development of wireless power transfer technology is getting more and more concern. This paper analyzed the wireless power transfer system through mutual inductance equivalent model and obtained the mathematical relationship between various parameters. It also analyzed the system's losses type and proposed some methods to reduce losses. In this paper, a new structure of transformer was used to design a set of WPT system it was lighter, smaller and had got a high efficiency which reached $94 \%$ through experiments.
\end{abstract}

Keywords-wireless power transfer; medium power; high efficiency; compensation topology

\section{INTRODUCTION}

Wireless power transfer is a new technology which uses electromagnetic induction, magnetic resonance, radiofrequency, etc. to achieve non-contact power transfer through the medium of air ${ }^{[1]}$. The emergence of this technology can compensate the shortcomings in traditional contact power transfer system. Such as the poor safety of power lines, trouble in equipment movement, unsightly environment, exposed power lines and easy to produce spark, etc ${ }^{[2]}$. Because of these obvious advantages compared to traditional contact power transfer system, WPT technology has expended the application prospects in the field of modern medicine, electric vehicles, military industry, industrial robots, etc. So the technology has also been listed as one "10 to lead the future of science and technology ".

In accordance with the principle of power transfer, the WPT technology mainly includes electromagnetic induction, electric field coupling, magnetic resonance and microwave technology. This paper used magnetic resonance technology, which was the Massachusetts Institute of Technology (MIT) announced in 2007, known as magnetic resonance induction or magnetically coupled resonance. Its principle was when the primary coil and secondary coil have same resonance frequency. The power can be transmitted from primary coil to secondary coil efficiently ${ }^{[3-4]}$.

Magnetic resonance wireless power transfer system uses alternating magnetic field space as its transmission medium, the intensity of the magnetic field determines the transmission performance of the entire system. Under the condition of the absence of core, spatial distribution of the magnetic field is dispersed, with lower mutual inductance, so that the system's performance has been hampered on a certain extent. Therefore, this system uses a new non-contact transformer, we add a new-type core based on the original transformer, and use a special winding way to improve transformer performance. The experiment had proved a higher transmission power and higher transmission efficiency after add the new-type structure of transformer into the system.

\section{THE STRUCTURE OF SYSTEM}

The configuration of wireless power transfer system shown in Figure 1, the system mainly included four parts: power supply circuit (high-frequency AC source), the power emission end, the power received end and the load end.

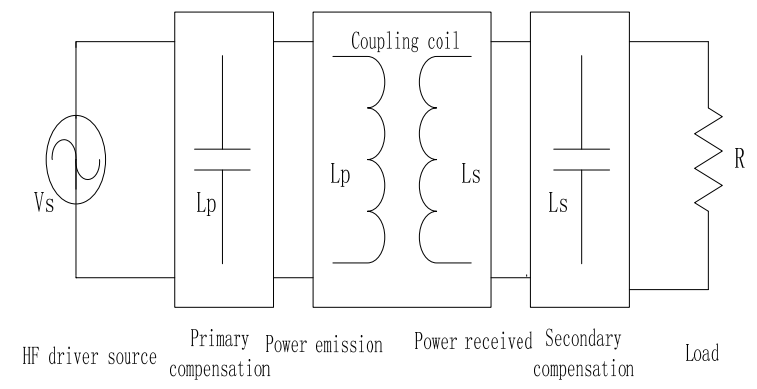

\section{FIGURE I. CONFIGURATION OF WPT}

The high-frequency AC source is the input of the WPT system, which got from high-frequency inverter circuit. The $L C$ resonant circuit is constituted by the compensation capacitors $C_{\mathrm{s}}, C_{\mathrm{p}}$, and primary coil inductance $L_{\mathrm{p}}$, secondary coil inductance $L_{\mathrm{s}}$. Make sure the resonant frequencies of primary and secondary were consistent, and the system will resonant under this frequency. In this way the power can transfer from the primary to the secondary side. After the secondary coil received the power, high-frequency alternating current will change into direct-current through the rectifier bridge. And the DC supplies the power for the load ${ }^{[5]}$.

\section{THE WPT SYSTEM’S ANALYSIS AND SIMULATION}

\section{A. Analysis of the System Efficiency}

To analyze the WPT system, people generally use mutual inductance equivalent model. This paper will analyze the system under the condition of series connection compensation topology both on primary and secondary sides. Other compensation topologies have corresponding analysis process according to the example in this paper. SS compensation topology is shown in Figure 2. The $V_{s}$ mean high-frequency driving source and $C_{\mathrm{p}} L_{\mathrm{p}}, C_{\mathrm{s}} L_{\mathrm{s}}$ are compensation 
capacitor and inductor on the primary and secondary sides, $r_{1}, r_{2}$ are the resistance on the primary and secondary coils.

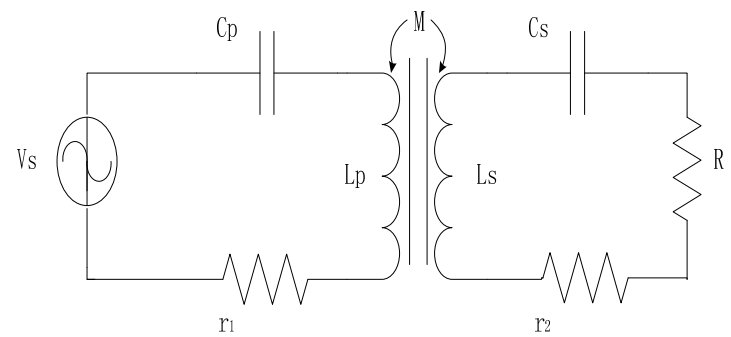

FIGURE II. SS COMPENSATION TOPOLOGY

The impedance of the secondary side is:

$$
\mathrm{Z}_{s}=j \omega L_{s}+\frac{1}{j \omega C_{s}}+R+r_{2}
$$

Therefore, according to mutual inductance equivalent model, $Z_{\mathrm{s}}$ mapped to the primary side is:

$$
Z_{r}=\frac{\omega^{2} M^{2}}{Z_{s}}
$$

In this case the impedance of primary side is:

$$
Z_{p}=j \omega L_{p}+\frac{1}{j \omega C_{p}}+r_{1}+Z_{r}
$$

When they have the same frequency:

$$
\omega_{0}=\frac{1}{\sqrt{L_{p} C_{p}}}=\frac{1}{\sqrt{\mathrm{L}_{\mathrm{s}} C_{s}}}
$$

Calculate primary current:

$$
I_{p}=\frac{U_{s}}{\frac{\omega^{2} M^{2}}{r_{2}+R}+r_{1}}
$$

Secondary current can be calculated also:

$$
I_{s}=\frac{j \omega M U_{s}}{\omega^{2} M^{2}+r_{1}\left(r_{2}+R\right)}
$$

So the system efficiency:

$$
\eta=\frac{P_{\text {OUT }}}{p_{I N}}=\frac{\omega^{2} M^{2} R}{\omega^{2} M^{2}\left(r_{2}+R\right)+r_{1}\left(r_{2}+R\right)^{2}}
$$

From the above equation, the level of efficiency of the system is related to the system operating frequency, mutual inductance, coil resistance, and load.

\section{B. Analysis of the System Loss}

From the above analysis shows that the consumption power in primary and secondary coils resistance are:

$$
\begin{aligned}
& P_{1}=I_{p}^{2} r_{1}=\left(\frac{U_{s}\left(r_{2}+R\right)}{\omega^{2} M^{2}+r_{1}\left(r_{2}+R\right)}\right)^{2} r_{1} \\
& P_{2}=I_{s}^{2} r_{2}=\left(\frac{j \omega M U_{s}}{\omega^{2} M^{2}+r_{1}\left(r_{2}+R\right)}\right)^{2} r_{2}
\end{aligned}
$$

The power loss of these two parts was consumed in the form of heat. Besides, the internal resistance of the primary and secondary coil, system equivalent resistance included the HF driver source's resistance, equivalent series resistance of compensation capacitor, and radiation resistance.

Litz wire can be used to reduce the coil resistance. It also reduces the skin effect and Proximity effects at high frequencies affected in the mean time.

The primary loss caused in high frequency driving source resistance is the loss in the high frequency power device. In this system, with the selected high frequency power device MOSFET tube, the loss mainly includes two aspects, the first one is MOS transistor conduction losses, on-resistance MOS tube is small in general, but large conduction current of the system makes this part of the loss can't be neglected. To reduce the conduction losses, the on-resistance of power tubes need to be rather smaller. Another one is switching losses of MOS transistor which is caused by delay of a MOS transistor switch. Due to large conduction current and voltage of MOS tube and a high switching frequency, so that this part of the loss is the major loss of the system, it can be reduced by choosing MOS transistor with lower switching delay or using a soft switch.

Toward specific system, there are specific requirements for the compensation capacitor. The features of CBB22 capacitor makes it be a preferable choice for a wireless power transfer system, such as high pressure for voltage, large current, high frequency and small equivalent series resistance.

Radiation resistance loss refers to the power coupling coil diffuse onto the medium of the air, it is related with shape of

\begin{tabular}{|c|c|c|c|c|}
\hline $\begin{array}{c}d \\
\mathrm{~mm}\end{array}$ & $\begin{array}{c}L_{p} \\
U_{H}\end{array}$ & $\begin{array}{c}L_{s} \\
U^{H} H\end{array}$ & $\begin{array}{l}L_{\text {close }} \\
\boldsymbol{U} H\end{array}$ & K \\
\hline 20 & 617 & 654 & 275 & 0.744 \\
\hline 50 & 460 & 472 & 366 & 0.453 \\
\hline 100 & 403 & 419 & 390 & 0.181 \\
\hline
\end{tabular}
the coil and the size of the current. Compared to other losses, it is relatively small, and therefore will not be discussed in detail in this paper.

\section{THE EXPERIMENTAL RESULTS AND ANALYSIS}

\section{A. New Transformer's Parameter Measurements}

This system uses an intelligent LCR tester LCR-8110G to measure relevant parameters, the result is shown in Table 1:

TABLE I. MEASURE DATA 
The $L_{\text {close }}$ in the table is the inductance when the primary coil is shorted and measure inductance in the secondary coil. The coupling coefficient $K$ is used to show the degree of couple for transformer, its maximum is 1, indicating complete couple. $K$ value is calculated as follows:

$$
\mathrm{K}=\sqrt{1-\frac{\mathrm{L}_{\text {close }}}{L_{p_{-} \text {open }}}}
$$

\section{B. Results and Analysis}

Experimental test system is shown in Figure 3:

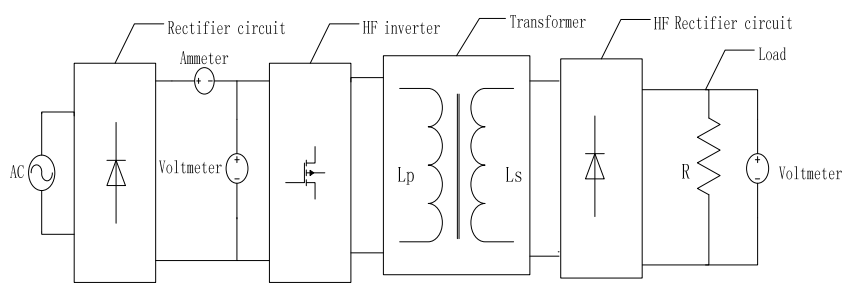

FIGURE III. SYSTEM DIAGRAM FOR MEASUREMENT

Measurement data is shown in Table 2:

TABLE II. MEASURE DATA

\begin{tabular}{lllll}
\hline \multicolumn{1}{c}{$V_{\text {in }} V$} & $V_{\text {out }}, V$ & $P_{\text {in }}, \mathrm{W}$ & $P_{\text {out },} \mathrm{W}$ & $\eta, \%$ \\
\hline 30 & 18.6 & 15.6 & 13.8 & 88.7 \\
70 & 43.2 & 79.7 & 73.2 & 91.8 \\
110 & 67.6 & 193.1 & 179.2 & 92.8 \\
170 & 104.5 & 455.2 & 428.2 & 94.1 \\
\hline \multicolumn{5}{r}{ The data is measured under the conditions of $60 \mathrm{~mm}$ gap, }
\end{tabular}

The data is measured under the conditions of $60 \mathrm{~mm}$ gap, the resonance frequency is $20 \mathrm{KHZ}$, and the load is $25.5 \mathrm{ohms}$.

\section{CONCLUSION}

This paper introduced the structure of the wireless power transfer system, and researched the related parameters and system efficiency through the theoretical analysis under compensation topology of SS. Analyzed the relationship between wireless power transfer system efficiency and mutual inductance, load value and the internal resistance of the coil. And use a new-type structure to design a transformer which is lighter and smaller than traditional transformer, and had got a higher efficiency which reached 94\% through the experiments.

\section{REFERENCES}

[1] WANG Chwei-sen, COVIC Grant A, STIELAU Oskar H. Power Transfer Capability and Bifurcation Phenomena of Loosely Coupled Inductive Power Transfer System [J]. IEEE Transaction on Industrial Electronic, 2004. 51 (1): 148-157.

[2] CHENG K W E. LU Y. Development of a Contactless Power Converter [J]. IEEE International Conference on Industrial Technology, 2002 (2): 786-791.

[3] Kurs A., Karalis A., Moffatt R., et al. Wireless power transfer via strongly coupled magnetic resonances [J]. science, 2007, 317(5834): 83-86.

[4] Chen Qingquan, Sun linqing. Present status and future trends of electric vehicles[J]. Science and Technology Review, 2005, 23 (4):24-28.

[5] Wu Ying, Yan Luguang, Huang Changgang, et al.Performance analysis of new contactless electrical energy transmission system[J]. Advanced
Technology of Electrical Engineering and Energy, 2003, 22(4):10-13,

43. 